\title{
Design of Cost-Optimal Passive Optical Networks for Small Cell Backhaul Using Installed Fibers [Invited]
}

\author{
Chathurika S. Ranaweera, Patrick P. Iannone, Kostas N. Oikonomou, \\ Kenneth C. Reichmann, and Rakesh K. Sinha
}

\begin{abstract}
With the recent popularity of mobile data devices, the demand for mobile data traffic has grown rapidly as never before. Hence, service providers are trying to come up with cost-effective solutions to battle this ever increasing demand for bandwidth in their cellular networks. Deployment of a denser heterogeneous network, with a large number of small cells, has been identified as an effective strategy not only to satisfy this unabated growth in mobile data traffic but also to facilitate ubiquitous wireless access. While the cost associated with on-site small cell equipment is low in comparison with a typical macro cell, deployment of small cells opens a new set of challenges, especially in relation to expenditures and capacity requirements associated with the backhaul. In this paper we discuss an efficient small cell backhauling strategy that leverages existing fiber resources in a cost-optimal manner. In particular, we formulate an optimization framework for planning a cost-minimized backhaul for a small cell network, which is based on the deployment of passive optical networks on top of the existing infrastructure. We demonstrate the effectiveness of our method by using our optimization framework to design a cost-optimal backhaul for a small portion of a realistic backhaul network. Our results show that in comparison to the typical point-to-point fiber backhauling approach, our technique can halve the costs associated with small cell backhaul deployment.
\end{abstract}

Index Terms-Mobile backhaul; Optimization; Passive optical network; Small cell network.

\section{INTRODUCTION}

$\mathbf{E}$ ver since the introduction of mobile communications, telecommunication service providers have been investigating different solutions to cope with the increasing bandwidth demand in their cellular networks. Until recently, technology upgrades combined with the deployment of additional macro-cell sites have enabled providers to stay ahead of demand. However, given the rapid growth of data usage due to the recent popularity of mobile data

Manuscript received May 2, 2013; revised August 2, 2013; accepted August 2, 2013; published September 26, 2013 (Doc. ID 189843).

C. S. Ranaweera is with the Department of Electrical and Electronic Engineering, The University of Melbourne, Australia (e-mail: c.ranaweera@ pgrad.unimelb.edu.au).

P. P. Iannone, K. N. Oikonomou, K. C. Reichmann, and R. K. Sinha are with AT\&T-Lab Research, 200 S. Laurel Avenue, Middletown, New Jersey 07748, USA

http://dx.doi.org/10.1364/JOCN.5.00A230 devices, such solutions are considered less effective today. The concept of heterogeneous cellular networks, i.e., the deployment of large numbers of small cells (microcellular network) to supplement the existing macro-cellular infrastructure, has recently emerged as a means to mitigate this supply-demand battle [1]. Despite its potential to satisfy future traffic growth [2], the costs associated with the deployment of a small cell network, while considerably less than those associated with macro cells, are significant due to the large expenditures associated with backhaul, real estate, etc. []]

Some large carriers have deployed fiber-based access networks, such as fiber-to-the-node (FTTN) networks [4], over a significant portion of their footprint to provide residential broadband access [5]. Spare fibers associated with these existing infrastructures can be leveraged to provide cost-effective backhaul for small cell network underlays to macro-cellular networks [6]. However, even when using previously installed dark fiber assets, care must be taken to utilize the fiber efficiently. Although such FTTN networks use a point-to-point (PTP) fiber topology, the dark fiber associated with these builds is a limited and valuable resource. Thus, opting for a PTP small cell backhaul architecture, while consistent with many new macro network backhaul deployments, can consume considerable available fiber resources, as it requires a pair of fibers for each connection. Furthermore, small cell underlay networks often consist of omnidirectional cells, which require a fraction of the bandwidth of macro cells, so a full gigabit Ethernet link is overkill.

This depletion of resources can be tempered by deploying passive optical networks (PONs) that share both fiber and central office (CO) terminal equipment. Standardized PON systems, such as gigabit PON (GPON) [7], use time division multiplexing to share a single $\mathrm{CO}$ transceiver and feeder fiber among a group of users, typically numbering 64 or fewer. The feeder fiber connects the $\mathrm{CO}$ to a splitter located in the vicinity of the user group. The splitter then connects to individual users over distribution fibers. GPON systems have a total downstream capacity of $2.5 \mathrm{~Gb} / \mathrm{s}$ and reach of $20 \mathrm{~km}$ or more (depending on the splitting ratio).

In this paper, we develop an optimization framework based on integer linear programming (ILP) [8], which can be used to plan PONs on top of existing fiber resources 
in order to cost-optimally backhaul a network of small cells. Given a set of planned small cell locations and details about existing infrastructure, our method determines the optimal fiber routes, the optimal locations for splitters and optical line terminals (OLTs), and the optimal number of PONs, for the purpose of most cost-effectively backhauling all the small cells. We demonstrate how our model can be used to plan a cost-optimal small cell backhaul network by applying it on a realistic backhaul network considering a range of different deployment scenarios. We show that for the scenarios we have considered, our proposed approach can save more than $50 \%$ of the deployment cost associated with small cell backhauling, in comparison to a typical PTP fiber backhauling approach.

The rest of the paper is organized as follows. Section II provides background on related work. Section III discusses different network architectures that use existing resources to backhaul small cells. In Section IV, an optimization framework is formulated that can be used to plan PONs on top of existing fiber infrastructure to backhaul small cells. Then Section V demonstrates how this optimization model can be used to design a cost-minimized small cell backhaul network for a small realistic backhaul network. A comparison between deployment costs of the optimized PON-based solutions under different deployment settings and the typical Ethernet-based PTP fiber backhauling approach is also presented in Section V. Finally, we conclude the paper in Section VI with a short summary of this study.

\section{Small Cell Deployment}

In addition to the obvious benefits, the deployment of small cells introduces a new set of challenges. In [9], small cell deployments are discussed in terms of energy efficiency and cost. Additionally, the authors use random matrix theory to analyze performance measures in small cell networks. Moreover, studies reported in [10] and [11] have given special attention to energy, which is an important constraint for next-generation network deployments. Another major aspect of small cell deployments in a heterogeneous environment is cochannel operation. In [1], the authors focus on addressing the issue of deploying small cells autonomically in a macro-cellular cochannel environment, for the universal mobile telecommunications system network.

To preserve all their benefits, small cells must be deployed cost-effectively. In particular, major cost contributors, such as those arising from backhaul, power, and real estate, need to be strategically reduced. To this end, we have explored the feasibility of locating small cells to coincide with existing fibered and powered network infrastructure [6]. Given such a set of planned small cell locations, this work attempts further cost reductions by optimizing the design of PONs constructed from the existing fiber infrastructure.

The optimal deployment of PONs for the purpose of providing fixed broadband access has been studied in [12-14], considering greenfield residential deployment scenarios.
These studies focused on determining optimal split ratios as well as the placement of fibers and splitters.

Our problem adds more complexity in comparison to the greenfield scenario, as it takes existing resources into account. An ILP-based optimization framework is used to plan the most economically efficient PON deployments constructed from existing dark fiber infrastructure, for the purpose of small cell backhaul. This technique outputs the dark fiber routes, the locations for splitters, locations for OLTs, and the number of PONs, to cost-optimally backhaul a given network of small cells. A summary of this work has been published in [15].

\section{BaCKHAul Network ArChitectures}

Leveraging existing FTTN infrastructure for small cell deployment is identified as an effective strategy to reduce the costs associated with the placement of small cells [6]. This section discusses architectures that can be used to backhaul small cells using existing FTTN resources. Figure 1 shows a distribution of FTTN remote terminals (RTs) and macro-cell sites in a realistic sample network used for the study described in [6]. In this scenario, each small cell is collocated with a FTTN RT. For example, the small cell's antenna and associated equipment can be placed on the light post/utility pole that is closest to each of the fiber-fed RTs. This strategy provides fiber backhaul, right of way, and powering for the deployment of small cells in an economical manner. Note that candidate small cell sites (red circles) are a subset of all fiber-fed RTs in the study area, consisting of only those that are separated from the nearest macro cell by at least a predetermined minimum distance (represented by light blue circles around the macro sites).

Figure 2(a) shows a typical PTP architecture that can be used to backhaul small cells using the existing FTTN infrastructure. As shown, each fiber-fed FTTN RT, at which the small cells are deployed, is connected to the CO through fiber access points (FAPs) by a pair of fibers. A FAP is essentially a location with access to the existing fiber, so that splices can be made between high fiber count feeder cables emanating from the $\mathrm{CO}$ and low fiber count distribution cables that terminate on RTs. For example, a FAP can be a manhole or a splicing box.

Dark fiber used in PTP architectures is a valuable resource and, as stated earlier, the consumption of those

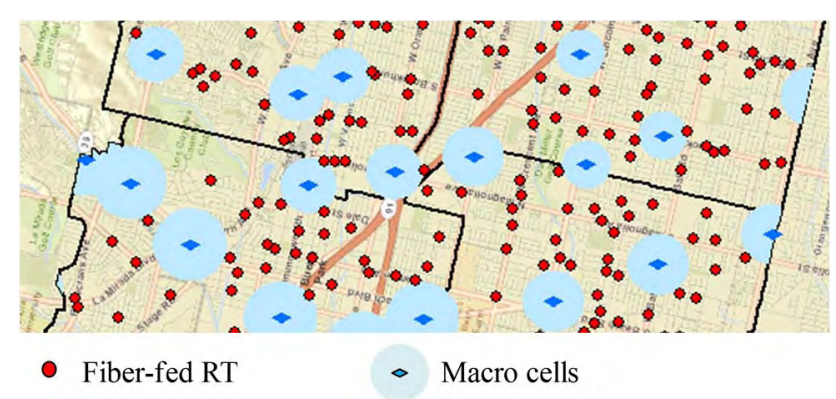

Fig. 1. Macro cells and FTTN fiber-fed RTs. 


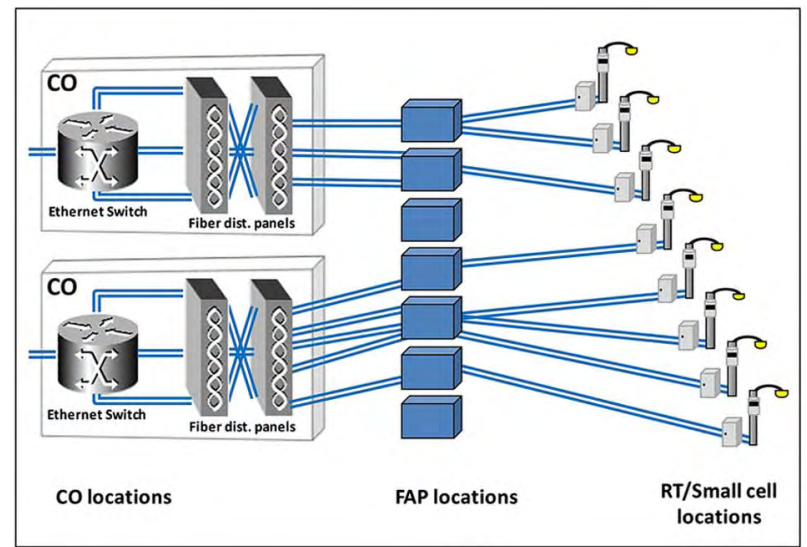

(a)

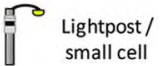

.7. Remote terminal (RT)

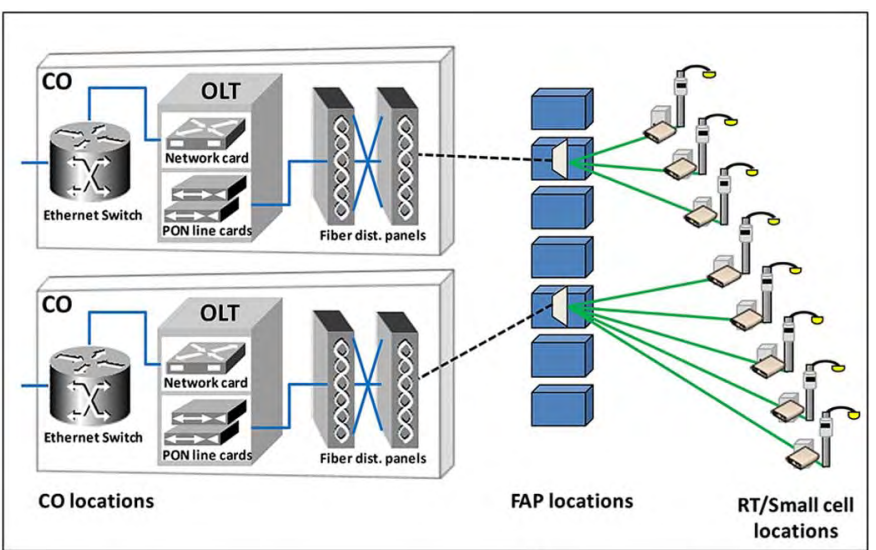

(b)

1 Passive splitter $=$ Fiber pai

- Single fiber --- feeder fiber - Distribution fiber

Optical network terminal (ONT)

Fig. 2. (a) Typical PTP backhaul architecture and (b) PON backhaul architecture.

fibers due to small cell backhauling can be reduced through the use of PONs. Figure 2(b) shows a PON-based small cell backhaul architecture that can be constructed from an existing dark fiber infrastructure. For such a network, new equipment needs to be installed, such as OLTs in the COs, splitters in selected preexisting FAPs, and optical network terminals (ONTs) in the RT locations. Optimal planning and placement of these new components in the existing fiber infrastructure is necessary in order to achieve the most economical deployment. This is discussed next.

\section{Optimization Framework}

In this section, we develop an optimization framework to minimize the total deployment costs of PON-based small cell backhaul that leverages existing infrastructure. First, the notation is introduced, then subsequent sections describe the components of the framework.

\section{A. Existing Infrastructure}

One of the key inputs to the optimization framework is the resources available in the existing network. The datasets representing these resources are defined as follows.

Let $\mathbf{C}$ denote the set of CO locations where OLTs can be placed. These are the locations where the access network is connected to the metro network. The serving area of one $\mathrm{CO}$ is referred to as a wire center. The set of existing fiber-fed FTTN RT locations that need to be backhauled using PON is denoted by $\mathbf{O}$. These are the locations where the small cells will be installed and therefore the ONTs are to be placed. Finally, the set of FAPs is denoted by M. These FAPs are the locations where the PON splitters can be installed or (in the case of the PTP network) where splices can be made to connect feeder fibers to distribution fibers. The main objective is to plan PON deployments to backhaul all of the locations in $\mathbf{O}$ using existing fiber resources, by choosing a subset of $\mathbf{M}$ for splitter installations and also choosing a subset of $\mathbf{C}$ for OLT installations, such that the total deployment cost is minimized.

\section{B. Cost Contributors}

The cost components of the PON-based backhaul network can be broadly classified into three categories: fiber costs, equipment costs, and labor costs.

Even dark fibers associated with an existing FTTN infrastructure incur a cost. This is due to the fact that previously installed dark fibers are valuable resources, and with the utilization of these fibers, the overall cable gets closer to exhaustion. Hence, a standard time-valueof-money approach is used to determine the cost of using existing fibers. Moreover, the fiber costs also depend on the type and length of fibers consumed by the small cell backhaul. Accordingly, the cost per unit length of distribution fiber is denoted by $\eta_{d}$ and the cost per unit length of feeder fiber by $\eta_{f}$.

Other than outside plant fiber, the equipment involved in such a deployment can be mainly categorized into three types based on where the equipment is installed or used in the network. These locations are in the RTs, in the COs, and in the FAPs.

At each RT location, an ONT must be installed to backhaul the collocated small cell. Let $\eta_{o}$ be the cost of an ONT.

In COs, the required equipment is OLTs, Ethernet switches, and indoor fiber connecting equipment. A typical setup inside the $\mathrm{CO}$ is illustrated in Fig. 2(b). The cost of the OLT depends on the required number of chassis, common equipment, and line cards, which in turn depends on the number of PONs connected to that CO. Typically, an OLT chassis supports several line cards and each line card supports multiple PONs. Let $n_{p}$ be the maximum number of PONs that can be supported by a line card. Let $\eta_{\mathrm{ch}}$ be the cost of an OLT chassis together with common equipment 
required for an OLT such as network cards, and let $\eta_{\text {olt }}$ be the cost of a line card.

Moreover, Ethernet switches are used to connect fibers from the access network to the metro network. Let $\eta_{e}$ be the cost of using a port in an Ethernet switch to connect a single fiber from the access side of the network to the metro network. There is also a fixed cost associated with terminating each feeder fiber at the CO. As shown in Fig. 2(b), these fiber distribution panels are required to make the connections from the outside plant fiber (feeder) to fiber jumpers that traverse the $\mathrm{CO}$ and terminate on the OLT shelf. We denote this fixed cost associated with one fiber connection at the $\mathrm{CO}$ by $\eta_{k}$.

In addition, there is a cost associated with the splitters installed in the FAPs. The cost of a splitter depends on the number of splits it supports and the type of enclosure in which it is installed. Therefore, the equipment-related cost involved in deploying splitters in the FAPs depends on how many enclosures and how many splitters will be installed at these selected FAPs. The cost of an enclosure together with the first splitter is denoted by $\eta_{s}$ and the cost of each additional splitter installed in this enclosure by $\eta_{a}$. The values of $\eta_{s}$ and $\eta_{a}$ vary depending on the splitting ratios.

Finally, in terms of labor costs, the splitter installations account for the bulk of the labor costs, since the major costs associated with fiber installations (e.g., trenching, etc.) do not apply directly in our case but are accounted for in the cost of the already installed fiber resources, as described previously. The labor costs associated with the splitter consist of a fixed cost to send personnel to the FAP location and to install the splitter(s) and enclosure, denoted by $\eta_{l}$, and the cost associated with splicing, which depends on the number of splices. The cost per splice is denoted by $\eta_{i}$. In addition, we also consider the labor costs associated with the work performed at the $\mathrm{CO}$, denoted by $\eta_{\mathrm{lc}}$. Table I summarizes all our cost parameters.

\section{Network Parameters}

In this subsection, we discuss various network requirements and parameters that depend on the deployment

TABLE I

Parameters Used for Cost Values

\begin{tabular}{lcl}
\hline Costs & Notation & \multicolumn{1}{c}{ Description of the Cost } \\
\hline Fiber & $\eta_{d}$ & Unit length of distribution fiber \\
& $\eta_{f}$ & Unit length of feeder fiber \\
Gear & $\eta_{o}$ & The cost of an ONT \\
& $\eta_{\text {ch }}$ & The OLT chassis and the common equipment \\
& $\eta_{\text {olt }}$ & The OLT line card \\
& $\eta_{e}$ & The Ethernet port usage \\
& $\eta_{k}$ & Fixed cost per fiber connection at the CO \\
& $\eta_{s}$ & The splitter enclosure together with \\
& & the first splitter \\
Labor & $\eta_{a}$ & An additional splitter \\
& $\eta_{l}$ & Splitter installation cost \\
& $\eta_{i}$ & Cost per splice \\
& $\eta_{\mathrm{lc}}$ & CO equipment installation \\
\hline
\end{tabular}

scenario and the type of equipment used. The locations of FAPs, COs, and small cells are used to determine the cost of connecting a small cell with a splitter and a splitter with the CO. Assuming a fixed cost per unit length of fiber, the "fiber-length" for each small cell-FAP pair and FAP-CO pair is precomputed. The fiber length of such a pair is the real distance along the existing fiber infrastructure. Moreover, if fiber cost is not proportional to its length, one can change the ILP to use the actual cost of fiber for each pair (instead of fiber length and cost per unit distance). Each of the COs, FAPs, and RTs is assigned a unique identifier. $l_{c, m}^{f}$ denotes the distance from the $c$ th $\mathrm{CO}$ to the $m$ th FAP, and $l_{m, o}^{d}$ denotes the distance from the $m$ th FAP to the oth ONT. Other parameters and sets are listed below:

- $n_{u}$ : Number of RT/ONT locations

- $n_{v}$ : Number of FAP locations

- $n_{w}$ : Number of CO locations

- $n_{p}$ : Number of PONs per line card

- $r$ : Split ratio

- $l_{\text {max }}$ : Maximum transmission distance

- C: The set of CO locations where $|\mathbf{C}|=n_{w}$

- M: The set of FAPs where $|\mathbf{M}|=n_{v}$

- O: The set of small cell/RT/ONT locations where $|\mathbf{O}|=n_{u}$.

\section{Variables}

The binary and integer decision variables used in the optimization framework are listed below:

- $f_{c, m}$ is a binary variable

$$
f_{c, m}=\left\{\begin{array}{cc}
1 ; & \begin{array}{c}
\text { if the } c^{\text {th }} \text { CO is connected to a } \\
\text { splitter at the } m^{\text {th }} \text { FAP }
\end{array} \\
0 ; & \text { otherwise }
\end{array}\right.
$$

- $\bar{f}_{c, m}$ is an integer variable: the number of connections between the $c$ th $\mathrm{CO}$ and the $m$ th FAP

- $d_{m, o}$ is a binary variable

$$
d_{m, o}=\left\{\begin{array}{cc}
1 ; & \text { if a splitter at the } m^{\text {th }} \mathrm{FAP} \text { is } \\
\text { connected to the } o^{\text {th }} \mathrm{ONU}
\end{array}\right.
$$

- $s_{m}$ is a binary variable

$$
s_{m}=\left\{\begin{array}{cc}
1 ; & \text { if at least one splitter is } \\
\text { placed at the } m^{\text {th }} \mathrm{FAP} \\
0 ; & \text { otherwise }
\end{array}\right.
$$

- $\bar{s}_{m}$ is an integer variable: the number of splitters installed at the $m$ th FAP location

- $\bar{x}_{c}$ is an integer variable: the number of line cards in the $c$ th CO location. 


\section{E. Optimization Model}

Our objective is to minimize the total deployment cost of PONs, which will be deployed on top of existing fiber infrastructure to backhaul small cells. The objective function of this optimization problem, given by Eq. (1), consists of different costs, as discussed in the previous subsection.

Objective function:

$$
\begin{aligned}
& \min \eta_{f} \sum_{c \in \mathbf{C}} \sum_{m \in \mathbf{M}} l_{c, m}^{f} \bar{f}_{c, m}+\eta_{d} \sum_{m \in \mathbf{M}} \sum_{o \in \mathbf{O}} l_{m, o}^{d} d_{m, o} \\
& +\sum_{c \in \mathbf{C}} \bar{x}_{c} \eta_{\mathrm{olt}}+n_{w}\left(\eta_{\mathrm{ch}}+\eta_{e}\right)+\sum_{m \in \mathbf{M}} \bar{s}_{m}\left(\eta_{k}\right) \\
& +\sum_{m \in \mathbf{M}} s_{m} \eta_{s}+\sum_{m \in \mathbf{M}}\left(\bar{s}_{m}-s_{m}\right) \eta_{a}+n_{u} \eta_{o}+n_{w} \eta_{\mathrm{lc}} \\
& +\sum_{m \in \mathbf{M}} s_{m} \eta_{l}+\left(n_{u}+\sum_{m \in \mathbf{M}} \bar{s}_{m}\right) \eta_{i} .
\end{aligned}
$$

In Eq. (1), the first cost component is the cost of fibers, which can be reduced by minimizing the length of both the feeder and the distribution fibers. The total cost arising from the feeder fiber usage is $\eta_{f} \sum_{c \in \mathbf{C}} \sum_{m \in \mathbf{M}} l_{c, m}^{f} \bar{f}_{c, m}$, while $\eta_{d} \sum_{m \in \mathbf{M}} \sum_{o \in \mathbf{O}} l_{m, o}^{d} d_{m, o}$ captures the total cost arising from the distribution fiber usage.

The total cost associated with OLT line cards is $\sum_{c \in \mathbf{C}} \bar{x}_{c} \eta_{\mathrm{olt}}$, while $n_{w}\left(\eta_{\mathrm{ch}}+\eta_{e}\right)$ represents the total cost associated with the OLT chassis, OLT common equipment, and the Ethernet switch. The total cost associated with feeder fiber connectivities at the $\mathrm{CO}$ is $\sum_{m \in \mathbf{M}} \bar{s}_{m}\left(\eta_{k}\right)$.

The total equipment cost arising from the first splitter and the enclosure installed at each and every selected splitter location is $\sum_{m \in \mathbf{M}} s_{m} \eta_{s}$, while the total cost of all additional splitters installed in selected splitter locations is represented by $\sum_{m \in \mathbf{M}}\left(\bar{s}_{m}-s_{m}\right) \eta_{a}$. Moreover, the total cost associated with all the ONTs deployed in the FTTN RT locations is captured by $n_{u} \eta_{o}$.

Furthermore, the term $n_{w} \eta_{\text {lc }}$ represents the total cost arising from labor at the CO locations. On the other hand, the total labor cost associated with all the splitter installations is represented as two components. That is, the total cost for sending personnel to selected FAPs to install splitters and enclosures is represented by $\sum_{m \in \mathbf{M}} s_{m} \eta_{l}$, while the total labor cost associated with splicing the distribution and feeder fibers at each and every splitter location is represented by $\left(n_{u}+\sum_{m \in \mathbf{M}} \bar{s}_{m}\right) \eta_{i}$.

Constraints:

The following constraints ensure that the resulting network design satisfies realistic network requirements:

1) Constraint on the feeder fiber connectivity:

$$
\sum_{c \in C} \bar{f}_{c, m}=\bar{s}_{m}, \quad \forall m \in \mathbf{M} .
$$

Typically in a PON, each splitter is connected to only one OLT. Equation (2) ensures this network requirement. It also ensures that the feeder fiber connections from the COs are only established with FAPs with splitters installed.

2) Constraints on the distribution fiber connectivity:

$$
\begin{gathered}
\sum_{m \in M} d_{m, o}=1, \quad \forall o \in \mathbf{O}, \\
d_{m, o} \leq s_{m}, \quad \forall m \in \mathbf{M}, \quad \forall o \in \mathbf{O} .
\end{gathered}
$$

Equation (3) ensures that the each ONT that we install at each of the RT locations is always connected to only one FAP. On the other hand, Eq. (4) makes sure that the FAP to which each ONT is connected has at least one splitter.

3) The relationship between $f_{c, m}$ and $\bar{f}_{c, m}$ :

$$
\begin{gathered}
\bar{f}_{c, m} \geq f_{c, m}, \quad \forall c \in \mathbf{C}, \quad \forall m \in \mathbf{M}, \\
\bar{f}_{c, m} / n_{u} \leq f_{c, m}, \quad \forall c \in \mathbf{C}, \quad \forall m \in \mathbf{M} .
\end{gathered}
$$

The binary variable $f_{c, m}$, which represents the connection between the $c$ th $\mathrm{CO}$ and the $m$ th FAP, and the integer variable $\bar{f}_{c, m}$, which represents the number of connections between the $c$ th $\mathrm{CO}$ and the $m$ th FAP, are related to each other by $f_{c, m}=\min \left(1, \bar{f}_{c, m}\right)$. This nonlinear relationship between these two decision variables is captured by two linear constraints given by Eqs. (ㅁ) and (6). In particular, Eq. (ㅁ) makes sure that $f_{c, m}$ is set to zero when $\bar{f}_{c, m}$ is zero, and Eq. ( $\left.\underline{6}\right)$ sets $f_{c, m}$ to 1 when $\bar{f}_{c, m}$ becomes a nonzero integer.

4) Constraint on the split ratio:

$$
\sum_{o \in O} d_{m, o} \leq r \bar{s}_{m}, \quad \forall m \in \mathbf{M} .
$$

The split ratio is an important parameter to determine the nominal available capacity per small cell in a PONbased backhaul network. The split ratio can be chosen as per the network requirements, such as capacity and reach. Typical split ratios in PON deployments are $1: 32,1: 16,1: 8$, and 1:4. The maximum number of ONTs in a PON is bounded by the split ratio of the splitter. For example, a PON equipped with a 1:16 splitter can support up to 16 ONTs. Equation (7) represents this constraint.

5) Constraint on the span of the PON:

$$
\begin{aligned}
& l_{c, m}^{f} f_{c, m}+l_{m, o}^{d} d_{m, o} \leq l_{\max }, \\
& \forall c \in \mathbf{C}, \quad \forall m \in \mathbf{M}, \quad \forall o \in \mathbf{O} .
\end{aligned}
$$

The span of the PON is determined by the maximum transmission distance, $l_{\max }$, which in turn depends on the power budget of the PON and the split ratio. For example, with a typical PON power budget, the spans of the PON become 20,26, and $32 \mathrm{~km}$ for split ratios $1: 32,1: 16$, and $1: 8$, respectively. The distance between an ONT and the OLT in a PON, that is, the total length 
of the feeder and distribution fiber, should not exceed $l_{\max }$. This constraint is captured in Eq. (8).

6) Relationship between $s_{m}$ and $\bar{s}_{m}$ :

$$
\begin{gathered}
\bar{s}_{m} \geq s_{m}, \quad \forall m \in \mathbf{M}, \\
\bar{s}_{m} / n_{u} \leq s_{m}, \quad \forall m \in \mathbf{M} .
\end{gathered}
$$

The nonlinear relationship between the binary variable $s_{m}$, which represents the installation of a splitter in the $m$ th FAP, and the integer variable $\bar{s}_{m}$, which represents the number of splitters installed in the $m$ th FAP, can be represented by $s_{m}=\min \left(1, \bar{s}_{m}\right)$. This nonlinear relationship is captured by the constraints given in Eqs. (9) and (10), in a manner analogous to Eqs. (ㅁ) and (ㅁ).

7) Constraint on the number of PONs per line card:

$$
\begin{gathered}
\bar{x}_{c} \geq\left(\sum_{m \in M} \bar{f}_{c, m}\right) / n_{p}, \quad \forall c \in \mathbf{C}, \\
\bar{x}_{c}<\left(\sum_{m \in M} \bar{f}_{c, m} / n_{p}\right)+1, \quad \forall c \in \mathbf{C} .
\end{gathered}
$$

A single line card placed in the OLT can only support up to $n_{p}$ PONs. Therefore, for every $n_{p}$ PONs, the OLT needs a new line card. The relationship between the required number of line cards for the $c$ th $\mathrm{CO}$ location, represented by the variable $\bar{x}_{c}$, and the number of PONs connected to the $c$ th $\mathrm{CO}$, represented by the variable $\bar{f}_{c, m}$, is $\bar{x}_{c}=\left\lceil\left(\sum_{m \in M} \bar{f}_{c, m} / n_{p}\right)\right\rceil$. This nonlinear relationship is represented by the two linear constraints given in Eqs. (11) and (12).

8) Bounds on decision variables:

$$
\begin{gathered}
\bar{s}_{m} \geq 0, \quad \forall m \in \mathbf{M}, \\
\bar{f}_{c, m} \geq 0, \quad \forall c \in \mathbf{C}, \quad \forall m \in \mathbf{M}, \\
\bar{x}_{c} \geq 0, \quad \forall c \in \mathbf{C}, \\
f_{c, m} \in\{0,1\}, \quad \forall c \in \mathbf{C}, \quad \forall m \in \mathbf{M}, \\
d_{m, o} \in\{0,1\}, \quad \forall m \in \mathbf{M}, \quad \forall o \in \mathbf{O}, \\
s_{m} \in\{0,1\}, \quad \forall m \in \mathbf{M} .
\end{gathered}
$$

Finally, the bounds on the integer variables used in our ILP are given in Eqs. (13)-(15), whereas the bounds on the binary decision variables are given in Eqs. (16) - (18).

\section{Model Evaluation}

We used the publicly available ILP solver (GLPK) [16]. In contrast to heuristic algorithms, which generate approximate solutions, ILP generates exact solutions, but the computation time can, in some instances, be substantially longer. Nevertheless, ILP is well suited to our problem for the following two reasons. First, since our optimization problem is involved in the network planning stage and we only need to solve it once for a given dataset, we do not have a stringent requirement on the execution time and a few hours of execution can be tolerated. Second, since the existing fiber network is deployed on a wire-center basis, the small cell backhaul network is designed wire center by wire center. In this case, the datasets that are input to the optimization framework are small enough to obtain solutions within an acceptable time. For example, for the dataset that is used for this demonstration, the optimal solution is achieved within a minute. It is necessary to point out, however, that a cost-optimal backhaul for small cells with millions of nodes will likely require a heuristic approach.

\section{A. Dataset}

Figure 3 shows the dataset used for our demonstration, which covers a CO serving area with a realistic FTTN infrastructure. This dataset consists of different fiber routes, a CO, FAPs, and RTs. Beginning at the CO (blue star), one can follow the brown solid lines that represent the fiber routes to a set of RTs, which are represented by red dots. The intended small cell locations are chosen to coincide with the RT locations, and hence ONTs are located at these RT locations. The FAPs, which are represented by green squares, are scattered across the fiber routes, and these are the potential locations for the splitter installations.

We consider GPON that supports up to 2.5 Gbps of downlink data rate [7] for our demonstration. Accordingly, the

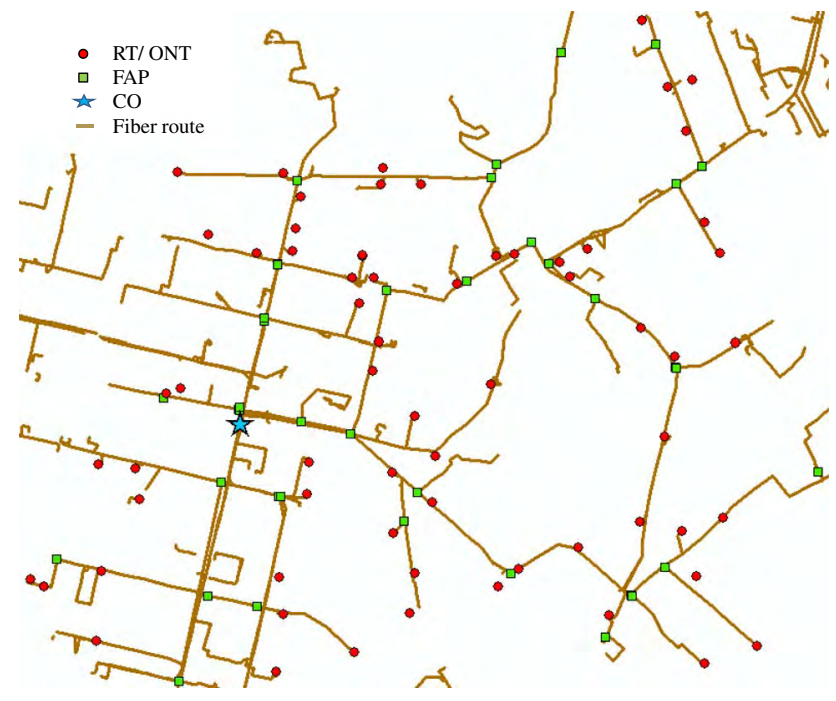

Fig. 3. Network dataset: FTTN infrastructure. 
TABLE II

Normalized Cost Values

\begin{tabular}{lc}
\hline Cost Component & Cost Value \\
\hline Feeder fiber/unit length & 8 \\
Distribution fiber/unit length & 25 \\
$1: 16$ - enclosure + 1 splitter & 18 \\
$1: 16$ - additional splitter & 9 \\
$1: 8$ - enclosure +1 splitter & 15 \\
$1: 8$ - additional splitter & 5 \\
$1: 4$ - enclosure +1 splitter & 15 \\
$1: 4$ - additional splitter & 2 \\
Ethernet switch/per port & 212 \\
Cost for fiber connection at CO & 28 \\
OLT chassis + common equipment & 472 \\
Line card; supports 4 PONs & 94 \\
ONT & 8 \\
Splitter installation & 16 \\
Splicing/per splice & 1 \\
Labor cost in CO & 20 \\
\hline
\end{tabular}

cost and performance parameters are selected based on the currently available GPON equipment. For other values such as fiber costs, fixed costs associated with feeder fiber connectivity at the COs, and costs of Ethernet switches, actual costs that are currently used in practice are considered. These costs are proprietary, so we summarize their values normalized with respect to the labor cost per splice in Table II.

In addition, the sets and parameters we have discussed in Section IV, i.e., $\mathbf{C}, \mathbf{M}, \mathbf{O}, n_{u}, n_{v}, n_{w}, n_{p}, r, l_{\max }, l_{c, m}^{f}$, and $l_{m, o}^{d}$, are also inputs. Outputs are 1) a subset of FAP locations where splitters should be installed, as well as the number of PONs at each one of those splitter locations, 2) the set of locations where OLTs should be installed, as well as the number of line cards at each of these OLT locations, and 3) connectivity between small cells and FAPs

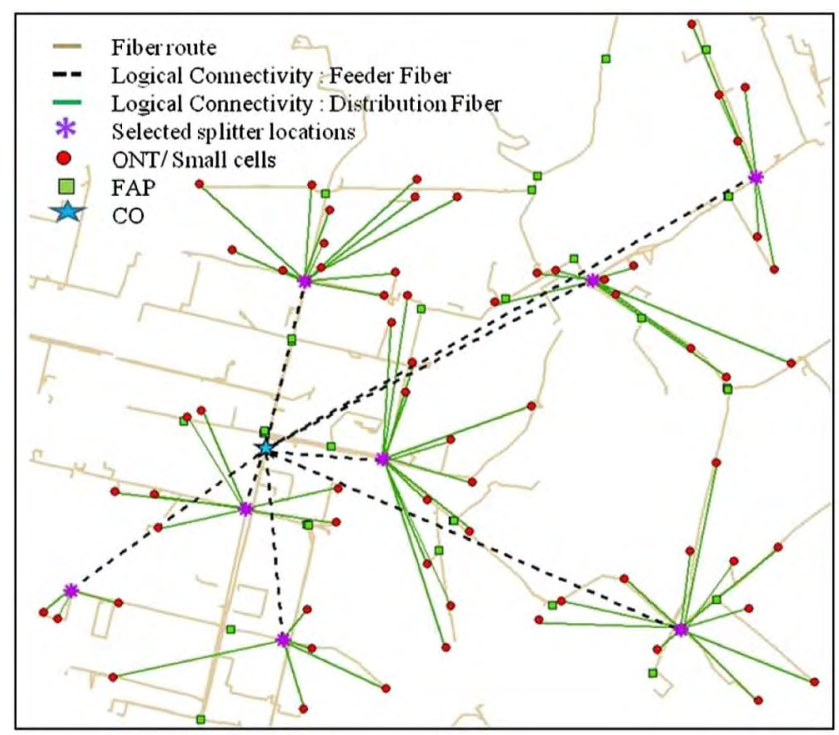

(a) and between FAPs and COs that suffices to backhaul the given set of small cells.

\section{B. Optimal Solutions}

We derive the optimal PON-based backhaul solution to this dataset for split ratios of $1: 16,1: 8$, and $1: 4$, which facilitate average backhaul capacities greater than $150 \mathrm{Mbps}$ per small cell. Moreover, for each deployment scenario considered, it is assumed that splitters with the same split ratio are used for all the PONs deployed within the test area. This is reasonable because most large carriers prefer to limit the variations among a given family of equipment in order to reduce costs associated with increased inventory, additional training, increased probability of craft error, etc.

Figures 4(a) and 4(b) show the backhaul solutions for the test area derived by our optimization framework when the maximum split ratio is $1: 16$ and $1: 8$, respectively. The asterisks represent the optimal splitter locations, and the black dotted lines represent the logical connectivities between the splitters and the CO. Green lines represent the logical connections between the small cell sites and the splitters. Careful inspection reveals that the optimal solution varies with the split ratio and the number of optimally selected splitter locations increases from 8 to 11 when the split ratio decreases.

\section{Cost Analysis}

In this subsection, we analyze the total deployment cost and the contribution of equipment, labor, and fiber costs in the optimal solution for a range of split ratios. In addition, we compare the total deployment cost of the

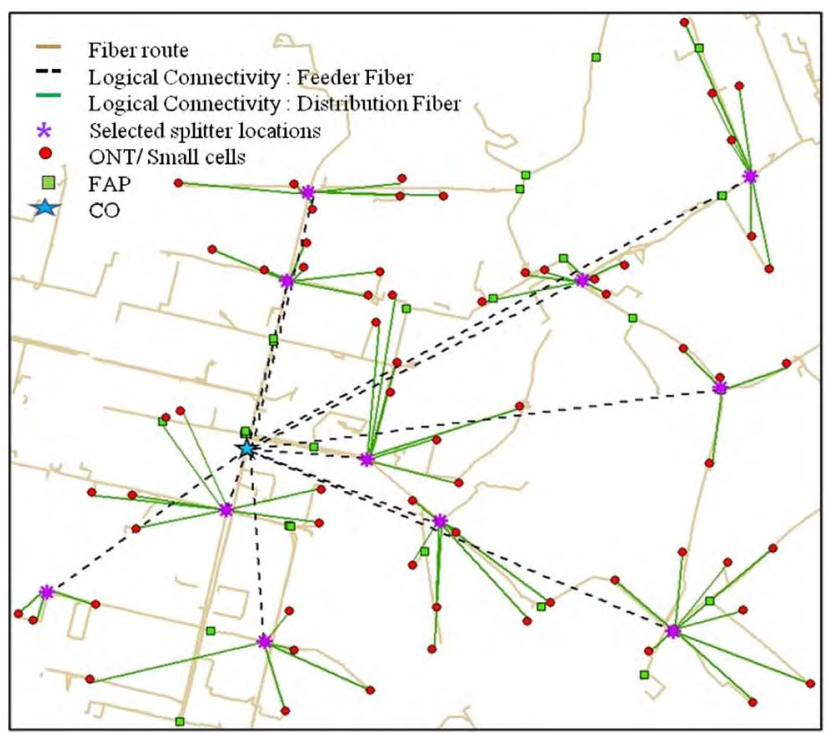

(b)

Fig. 4. Optimal solution when the split ratio is (a) $1: 16$ and (b) 1:8. 
PON-based solution with that of the PTP small cell backhaul solutions.

As was discussed previously, typical PTP backhaul architectures use a pair of fibers for each connection. Apart from this strategy, we also considered a PTP backhaul scenario where a single fiber is used per connection. This deployment strategy will be referred to as PTP-single, whereas the typical strategy in which a pair of fibers is used for each connection is referred to as PTP or typical PTP.

In order to evaluate the total cost of the PTP backhaul approach, it is assumed that the RTs are connected to their nearest FAPs, and subsequently to the COs, by the same types of fiber cabling (distribution and feeder) that are used for the PON case. Consequently, the estimate of total cost of the PTP backhaul approach is conservative, since a large portion of each PTP link is assumed to consist of low cost feeder fibers. In addition to the fiber, the remainder of the costs involved in the PTP backhaul deployments are from the equipment at the $\mathrm{CO}$ and the labor. The major difference between the PON deployment and PTP at the CO is that PTP connections do not require an OLT and its corresponding connections [see Figs. 2(a) and 2(b)]. Moreover, labor costs considered in the PTP deployment are for the work performed in the $\mathrm{CO}$ and fiber splicing required in the FAP to connect the distribution fiber(s) to the feeder(s). (The corresponding calculation in [15] mistakenly omitted some labor costs for the PTP case.)

Figure 5 compares the total deployment costs of optimized PON-based and PTP-based backhaul approaches, with the costs normalized with respect to the total deployment cost of the PTP solution so that the total PTP deployment cost is 100. It also illustrates the fractional contributions of equipment, labor, and fiber costs to the total for each of the approaches. The major cost contributor in the optimized PON-based solution is the equipment, while the fiber cost dominates the typical PTP backhaul solution. However, in the PTP-single deployment scenario, the equipment is the major cost contributor, because it only uses a single fiber for backhauling.

As shown in Fig. 5 for the optimized PON-based solution, the equipment cost increases as the split ratio decreases.

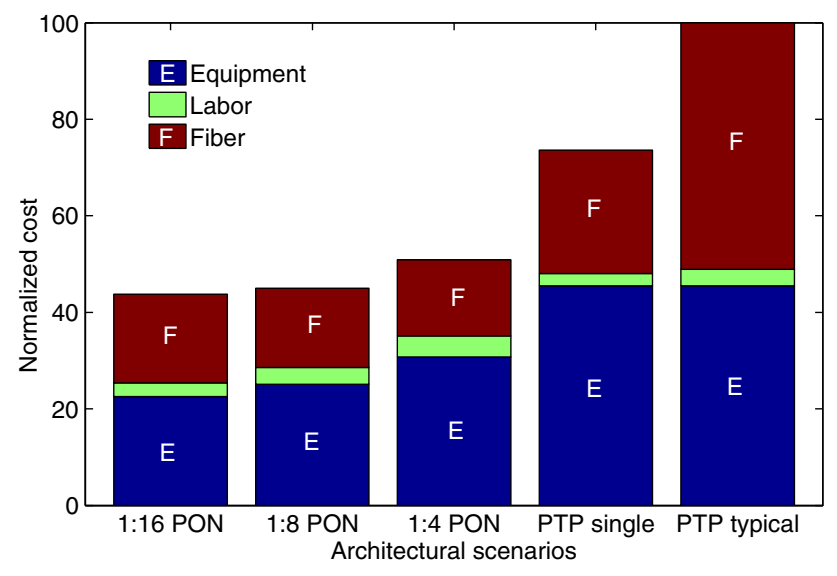

Fig. 5. Total deployment cost.
This is because when the split ratio decreases, the number of PONs needed to backhaul a given number of small cells increases. As a result, increased numbers of splitters and splitter enclosures, as well as the line cards in the OLTs, need to be deployed. This increases the total deployment cost. On the other hand, the fiber cost of the PON-based backhaul decreases when the split ratio decreases. This can be explained using Fig. 6, which shows the lengths (normalized) of distribution and feeder fibers used in the optimal PON-based backhaul solutions. When the split ratio decreases, the total length of the distribution fiber decreases, while the total length of feeder fiber increases as a result of increased numbers of optimally placed splitters. Since the cost of distribution fiber is significantly higher than that of the feeder fiber, when the total length of distribution fiber decreases, the total cost of fiber is also reduced.

Note in Fig. 5 that both the fiber and the equipment costs in all of the optimized PON-based backhaul solutions are considerably lower in comparison to the PTP backhaul solutions. In addition, the labor costs in both the PON-based and PTP backhaul solutions comprise only a small fraction of the deployment cost of each of the architectures due to the usage of the existing fiber infrastructure.

Figure 7 shows the percentages of cost savings that can be achieved by using the cost-optimal PON-based backhaul solution in place of typical PTP and PTP-single backhaul solutions. Therefore, a significant reduction in the equipment and fiber costs using the PON-based backhauling solution is demonstrated. Overall, using our optimization framework, the total deployment cost to backhaul with a typical two-fiber PTP solution can be reduced by more than half using the PON-based backhaul approach.

\section{Splitter Consolidation}

Large carriers often prefer to reduce the number of outof-office equipment sites in order to reduce operational and management costs. The collocation of splitters is one of the potential strategies that has been used to satisfy this requirement. In this section, we revisit the original

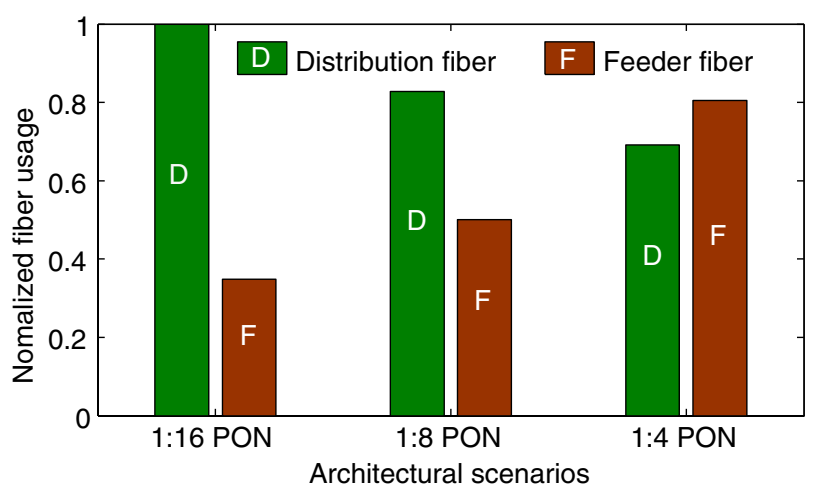

Fig. 6. Usage of fiber in optimized PON-based backhaul deployment. 


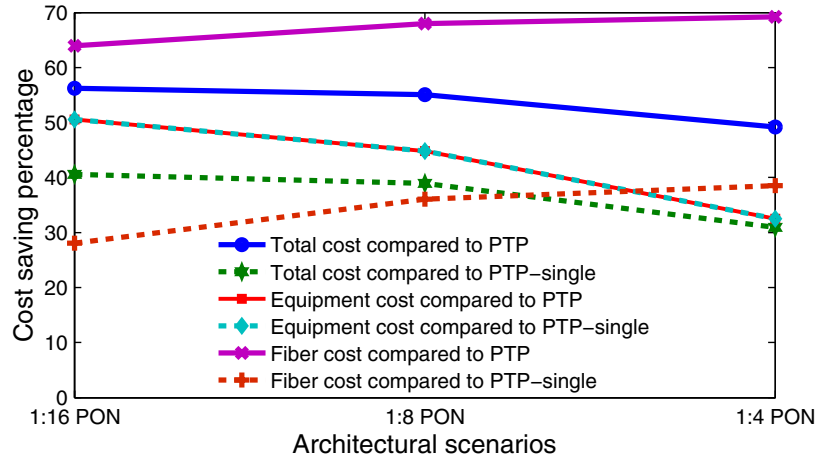

Fig. 7. Cost saving percentages.

optimization problem, taking splitter consolidation into account. In particular, we evaluate the total cost of the optimized PON-based solutions as a function of the number of maximum allowable splitter locations.

In order to capture the splitter consolidation requirement, the ILP is modified by adding another constraint that represents the maximum allowable number of splitter locations for the deployment. This constraint is

$$
\sum_{m \in M} s_{m} \leq n_{\mathrm{sl}}
$$

where $n_{\mathrm{sl}}$ is the maximum allowable number of splitter locations for the deployment.

Figure 8 shows the variation of the total deployment costs of different PON-based backhaul solutions as a function of the number of allowable splitter locations, $n_{\mathrm{sl}}$. Again, all the costs are normalized with respect to the total deployment cost of a typical PTP deployment scenario. As can be seen, the total deployment costs of optimal PONbased backhaul approaches decrease when $n_{\mathrm{sl}}$ increases. In particular, note that when splitters are deployed only in two locations, the total deployment cost in each of the scenarios nearly doubles in comparison to the relevant case when there is no restriction on the number of splitter locations. However, all of these cost values are still lower than the total deployment cost of a typical PTP solution. It is therefore evident that there is a quantifiable cost associated with the splitter consolidation requirement. Although

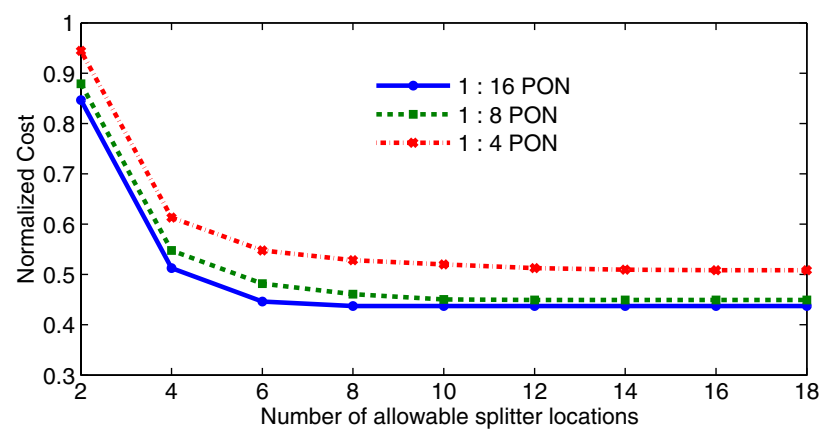

Fig. 8. Splitter consolidation. it is beyond the scope of this paper, carriers must weigh this cost against operational and maintenance savings derived from splitter consolidation.

\section{ConcLusion}

Leveraging existing infrastructures is one of the strategies that can be used to minimize the costs associated with small cell deployments. To further reduce the backhaul costs of small cells, we can deploy PONs constructed from existing fiber infrastructure. In this paper, we developed an optimization framework to plan cost-optimal deployments of PONs using existing fiber infrastructure. Such a method incorporates major cost contributors such as fiber, equipment, and labor and minimizes the total deployment cost by determining the best fiber routes, the optimal locations for splitters, and the optimal number of PONs that need to be used to backhaul a given set of small cells. As a concrete example, we planned a PONbased backhaul network for small cells located within a realistic backhaul network and showed that the cost of small cell backhauling can be reduced to half that of the typical PTP backhaul approach. Although applied to the problem of small cell backhaul network design, this method is broadly applicable to the design of optimized tree and branch architectures based on preexisting dark fiber infrastructure.

\section{REFERENCES}

[1] H. Claussen, L. Ho, T. W. Lester, and L. G. Samuel, "An overview of the femtocell concept," Bell Labs Tech. J., vol. 13, pp. 221-245, 2008.

[2] Cisco, "Cisco visual networking index: Global mobile data traffic forecast update, 2012-2017," 2013 [Online]. Available: http://www.cisco.com/.

[3] Next Generation Mobile Networks (NGNM), "Small cell backhaul requirements," White Paper, 2012 [Online]. Available: http://www.ngmn.org.

[4] M. Esfandiari, "Reliability evaluation of SBC's fiber to the node network," in Optical Fiber Communication Conf. and the Nat. Fiber Optic Engineers Conf. (OFC/NFOEC), 2006, paper NWD1.

[5] F. J. Effenberger, K. McCammon, and V. O'Byrne, "Cost minimization planning for passive optical networks," J. Opt. Netw., vol. 6, no. 7, pp. 808-8180, 2007.

[6] P. Iannone, K. Reichmann, C. Ranaweera, and M. Resende, "A small cell augmentation to a wireless network leveraging fiber-to-the-node access infrastructure for backhaul and power," in Optical Fiber Communication Conf., 2013, paper OTu2E.2.

[7] "Gigabit-capable passive optical networks (GPON): General characteristics," ITU-T Recommendation G.984.1, 2008.

[8] A. Schrijver, Theory of Linear and Integer Programming. Wiley, 1998.

[9] J. Hoydis, M. Kobayashi, and M. Debbah, "Green small-cell networks," IEEE Veh. Technol. Mag., vol. 6, no. 1, pp. 37-43, 2011.

[10] O. Arnold, F. Richter, G. Fettweis, and O. Blume, "Power consumption modeling of different base station types in 
heterogeneous cellular networks," in IEEE Future Network and Mobile Summit, Florence, 2010.

[11] B. Badic, T. O'Farrrell, P. Loskot, and J. He, "Energy efficient radio access architectures for green radio: Large versus small cell size deployment," in IEEE 70th Vehicular Technology Conf. Fall (VTC 2009-Fall), Anchorage, AK, 2009.

[12] J. Li and G. Shen, "Cost minimization planning for passive optical networks," in Optical Fiber Communication Conf. and the Nat. Fiber Optic Engineers Conf. (OFC/NFOEC), 2008, paper NThD1.

[13] A. Agata and K. Nishimura, "Suboptimal PON network designing algorithm for minimizing deployment cost of optical fiber cables," in Optical Network Design and Modeling (ONDM), Colchester, UK, 2012.

[14] S. U. Khan, "Heuristics-based PON deployment, " IEEE Commun. Lett., vol. 9, no. 9, pp. 847-849, 2005.

[15] C. Ranaweera, P. Iannone, K. Oikonomou, K. Reichmann, and R. Sinha, "Cost optimization of fiber deployment for small cell backhaul," in Optical Fiber Communication Conf. and the Nat. Fiber Optic Engineers Conf. (OFC/NFOEC), 2013, paper NTH3F.2.

[16] GNU, "GNU Linear Programming Kit," version glpk-4.47.1, Sept. 2011 [Online]. Available: http://www.gnu.org/software/ glpk/.

Chathurika S. Ranaweera received her B.Sc. degree in electrical and electronic engineering from The University of Peradeniya, Sri Lanka, in 2007. She is currently pursuing her Ph.D. degree in electrical and electronic engineering at The University of Melbourne, Australia. Her research interests include optical and wireless access networks, optical-wireless convergence, small-cell backhauling, and network optimization.

Patrick P. Iannone received his B.S. degree from Columbia University in 1984 and his Ph.D. degree from Princeton University in 1994. He joined AT\&T Bell Laboratories (later AT\&T Labs) in 1985, where he did research in multibeam microwave antennas, semiconductor laser nonlinearities, subcarrier-multiplexed lightwave communication systems and dense wavelength division multiplexed (DWDM) systems. Over the past several years, he has worked on WDM optical networks and subsystems for residential access, business access, metro transport, and wireless backhaul. He holds 40 patents and is a Fellow of the IEEE.

Kostas N. Oikonomou is a principal member of the technical staff at AT\&T Labs Research. His research interests include probabilistic and combinatorial modeling and analysis, with an emphasis on networks. He has a Ph.D. degree in electrical engineering from the University of Minnesota, and is a member of the IEEE.

Kenneth C. Reichmann is a Principal Member of Technical Staff in the Optical Systems Research department at AT\&T LabsResearch, concentrating on mobile backhaul and optical access technologies. He is a Senior Member of the IEEE.

Rakesh K. Sinha is a lead member of the technical staff at AT\&T Labs-Research. He has broad research interests in the areas of network architecture, design, and optimization. He received his B.Tech. degree from I.I.T. Kanpur, India, and his Ph.D. degree from the University of Washington, Seattle, both in computer science. 